\title{
Real-World User Experiences with a Digital Pill System to Measure PrEP Adherence: Perspectives from MSM with Substance Use
}

\author{
Peter R. Chai ${ }^{1,2,3,4}$. Georgia R. Goodman ${ }^{1,2,5}$. Olivia Bronzi ${ }^{2} \cdot$ Gerard Gonzales $^{2}$ - Alejandro Baez ${ }^{2}$. \\ Maria J. Bustamante ${ }^{2}$. Jesse Najarro ${ }^{2}$. Yassir Mohamed ${ }^{2} \cdot$ Matthew C. Sullivan $^{2,5} \cdot$ Kenneth H. Mayer ${ }^{2,6}$. \\ Edward W. Boyer ${ }^{1,2} \cdot$ Conall O'Cleirigh $^{2,5} \cdot$ Rochelle K. Rosen $^{7,8}$
}

Accepted: 15 January 2022 / Published online: 28 January 2022

(c) The Author(s), under exclusive licence to Springer Science+Business Media, LLC, part of Springer Nature 2022

\begin{abstract}
Once-daily oral pre-exposure prophylaxis (PrEP) is highly effective for preventing HIV transmission, but adherence can be challenging for men who have sex with men (MSM) who use substances. A novel method for directly measuring ingestion events is a digital pill system (DPS), which comprises an ingestible radiofrequency emitter that signals a wearable Reader device upon PrEP ingestion, relaying ingestion data to a wearable Reader device and then to a smartphone application. Qualitative interviews were conducted with $15 \mathrm{MSM}$ with non-alcohol substance use following an open-label pilot demonstration trial involving use of the DPS to measure PrEP adherence for 90 days. The purpose of this qualitative investigation was to understand overall user experiences and potential barriers and facilitators to using the DPS to measure PrEP adherence among MSM. The DPS was largely perceived as acceptable, novel, and valuable, with most participants reporting that the system was easily integrated into their daily routines. Technological and design factors, especially related to the wearable Reader, impacted participants' interest in using the technology long-term; several suggested improvements were discussed. Trial Registration ClinicalTrials.gov: NCT03842436.
\end{abstract}

Keywords Digital pill system · Ingestible sensors · PrEP · HIV prevention · Adherence

Peter R. Chai

pchai@bwh.harvard.edu

1 Department of Emergency Medicine, Brigham and Women's Hospital, 75 Francis St., Boston, MA 02411, USA

2 The Fenway Institute, Fenway Health, Boston, MA, USA

3 The Koch Institute for Integrated Cancer Research, Massachusetts Institute of Technology, Cambridge, MA, USA

4 Department of Psychosocial Oncology and Palliative Care, Dana Farber Cancer Institute, Boston, MA, USA

5 Department of Psychiatry, Massachusetts General Hospital, Boston, MA, USA

6 Infectious Diseases, Beth Israel Deaconess Medical Center/Harvard Medical School, Boston, MA, USA

7 Center for Behavioral and Preventive Medicine, The Miriam Hospital, Providence, RI, USA

8 Department of Behavioral and Social Sciences, Brown University School of Public Health, Providence, RI, USA

\section{Introduction}

Effective methods for measuring adherence to oral preexposure prophylaxis (PrEP) for HIV prevention are needed to end the HIV epidemic. When taken as directed, oncedaily tenofovir disoproxil fumarate/emtricitabine (TDF/ FTC) or tenofovir alafenamide/emtricitabine (TAF/FTC) have been demonstrated to be $99 \%$ efficacious in preventing new HIV acquisition [1]. However, the effectiveness of oral PrEP remains closely linked to adherence [2]. Maintaining consistent adherence to PrEP can be challenging, especially in the context of changes in routine, substance use, PrEP-related stigma, and mental health comorbidities [3]. Unfortunately, there is no gold standard for measurement of adherence behavior; most currently available techniques for measuring adherence depend on indirect methods (e.g. selfreported adherence, measuring drug concentrations), which vary in reliability $[4,5]$.

Men who have sex with men (MSM) who use substances are at risk for PrEP nonadherence and potential HIV infection via risky sexual behaviors like condomless anal sex or 
injection drug use; the measurement of PrEP adherence and accompanying support for maintaining PrEP adherence is therefore especially important in this population $[6,7]$. A recent study found that the use of psychostimulants (e.g., ketamine, ecstasy, gamma-hydroxybutyrate [GHB], cocaine, and methamphetamine) was a significant predictor of sameday missed doses of PrEP among MSM [8]. Additionally, in the same study, the likelihood of PrEP nonadherence increased among individuals who used club drugs by $55 \%$ for same-day doses, and $60 \%$ for doses on the following day [8].

One novel technique for assessing PrEP adherence is a digital pill system (DPS) $[9,10]$. A DPS incorporates (1) an ingestible sensor embedded within a gelatin capsule, (2) a medication of interest (e.g., PrEP), and (3) a wearable Reader device that acts as a receiver (Fig. 1). The gelatin capsule containing the ingestible sensor overencapsulates the medication, forming a "digital pill." Following ingestion, the digital pill dissolves in the stomach upon interaction with gastric acid, which releases the medication and activates the sensor. The sensor then wirelessly emits a signal to the wearable Reader via Bluetooth Low Energy (BLE). The Reader captures, stores, and then transmits the timestamped ingestion data to the user's smartphone, as well as to an online provider-facing interface through a cloud-based server. Real-time ingestion data is then accessible by both patients and healthcare providers and can be used to track adherence patterns and monitor nonadherence. Unlike other direct methods of adherence measurement (e.g., biological measures), the innovativeness of the DPS derives from its ability to directly measure and transmit medication adherence information in real-time. This real-time adherence data from the DPS is granular and can delineate both discrete episodes of suboptimal adherence, as well as patterns proximal to frank nonadherence. By measuring adherence behavior in real-time, the DPS is able to detect discontinuations of PrEP in times when individuals may inadvertently still be at heightened sexual risk, Moreover, the DPS also provides researchers and clinicians an opportunity to better recognize and support individuals' transitions from continuous PrEP adherence to alternative PrEP dosing strategies, such as event-driven PrEP [11, 12].

Several prior investigations have deployed a DPS to measure adherence, including in the evaluation of adherence to antidiabetic agents, antiretroviral therapy (ART), tuberculosis treatment, and antipsychotic medication for schizophrenia $[6,7,13,14]$. In a pilot study of fifteen people living with HIV (PLWH), reported experiences with the DPS were mixed [6]. Many participants perceived digital pills to be useful for the management of ART adherence, but design issues represented an inconvenience and barrier to use [6]. Users in other studies reported generally positive overall

\section{How the ID-Cap ${ }^{\text {th }}$ System Works}

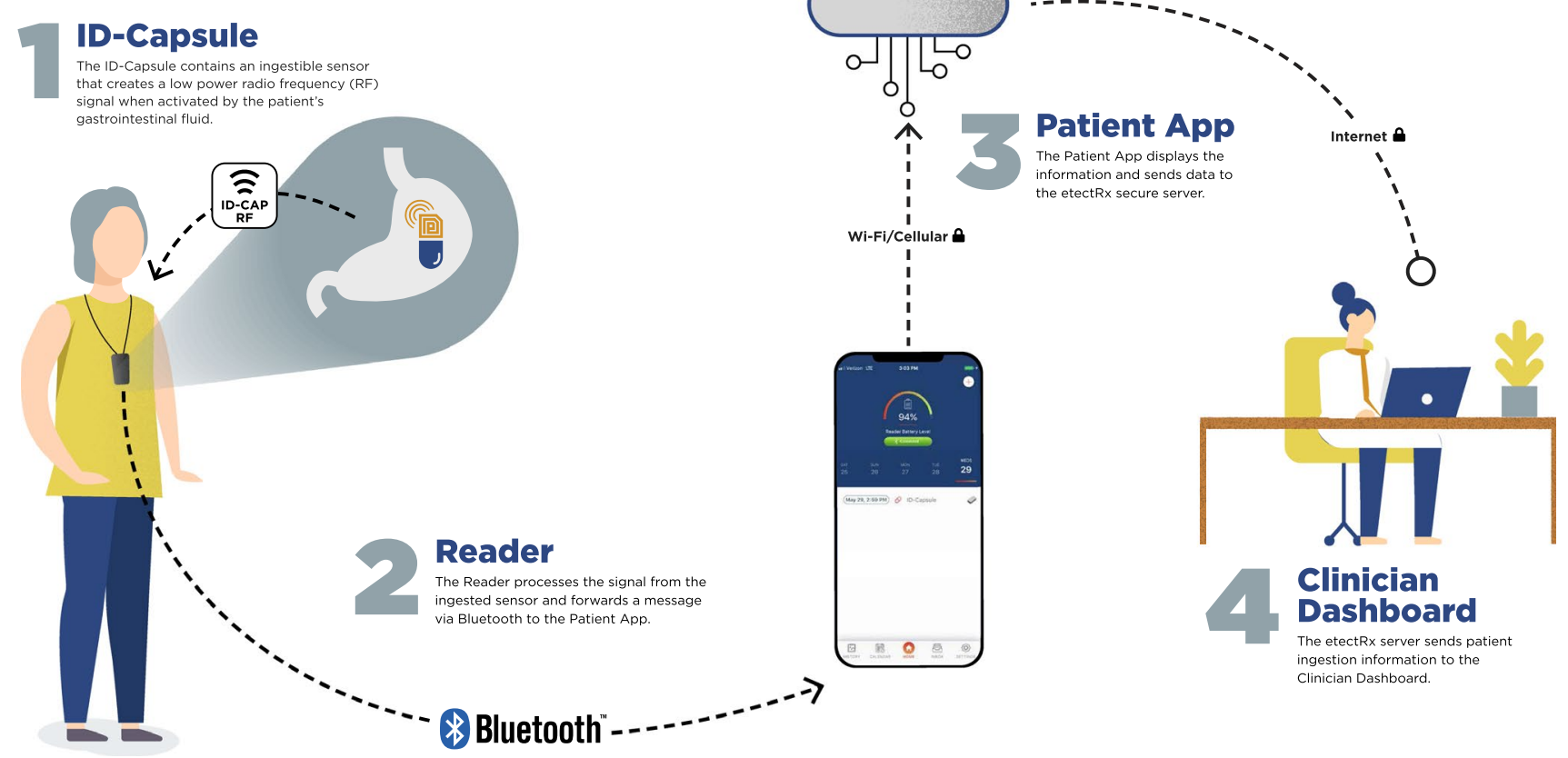

Fig. 1 Overview of digital pill system (DPS). The DPS includes an ingestible sensor within a gelatin capsule that over-encapsulates a medication. When ingested, it is activated by gastric fluid, broadcast- ing a radiofrequency signal that is acquired by a wearable Reader device and relayed to a smartphone which can display and transmit real-time adherence metrics. Image courtesy of etectRx 
experiences with the DPS; for example, $78 \%$ of patients with schizophrenia reported satisfaction after five weeks of independently using digital pills with their antipsychotics [13]. Additionally, a previous qualitative investigation conducted by our research team explored the feasibility and acceptability of using a DPS to measure PrEP adherence among thirty HIV-negative MSM who use non-alcohol substances. Our findings indicated that MSM were accepting of the DPS, described the technology as innovative and valuable, and largely expressed a willingness to use it for adherence measurement [9].

We conducted an open-label, pilot demonstration trial of fifteen MSM with non-alcohol substance use who used the DPS to measure PrEP adherence over a 3-month period. The overarching aims of this investigation were to assess the feasibility and acceptability of the DPS to measure realtime PrEP adherence in this population. At the conclusion of the study, we conducted individual, semi-structured, qualitative interviews to explore participants' lived experiences using the DPS. The feasibility data from this investigation are reported elsewhere; here, we report the emergent qualitative themes from these interviews in the context of overall acceptability of the DPS for measuring adherence to PrEP.

\section{Methods}

\section{Participants}

Sixteen participants enrolled in the pilot demonstration trial (NCT03842436). All participants were: (1) age 18 or older; (2) cisgender MSM; (3) self-reporting substance use other than alcohol during the past six months; (4) currently prescribed once-daily TDF/FTC as PrEP; and (5) had qualifying laboratory tests for PrEP (i.e., negative rapid HIV test, creatinine clearance $\geq 60 \mathrm{ml} / \mathrm{min}$, prior hepatitis B immunization, screening for sexually transmitted infections [STIs]). Exclusion criteria included: (1) non-English-speaking; (2) self-reporting living with HIV; (3) transgender; (4) estimated creatinine clearance $<60 \mathrm{ml} / \mathrm{min}$; (5) receiving active hepatitis B treatment; (6) taking proton pump inhibitors; (7) history of Crohn's disease or ulcerative colitis; (8) history of bowel surgery, gastric bypass, or bowel stricture; (9) history of GI malignancy or radiation to the abdomen; (10) not owning a smartphone; or (11) being unable or unwilling to ingest the digital pill. The study was approved by the Fenway Community Health Institutional Review Board (IRB), and was conducted between March 2019 and April 2020.

\section{Procedures}

Participants were recruited via social media advertising and community outreach throughout the Boston metropolitan area. Potential participants were pre-screened by a member of the study team; individuals who met preliminary eligibility criteria during the pre-screening were scheduled to attend an in-person screening visit to confirm eligibility.

Eligible participants attended a baseline study visit, where they completed a brief quantitative assessment, followed by a structured training session on how to operate the DPS [15]. Participants then received a 30-day supply of digital PrEP pills and demonstrated their competence with the technology by ingesting their first digital pill under observation by the study team. Two additional 30-day refills of digital PrEP pills were provided during follow-up study visits at months one and two. Participants used the DPS to measure their PrEP adherence for a total of 90 days, and were instructed to ingest one digital PrEP pill daily.

At the final study visit at month three, participants completed an individual, semi-structured, qualitative interview to provide feedback on their experiences using the DPS. Interviews were conducted by the principal investigator and/or a member of the study team trained in qualitative interviewing techniques. Remuneration was provided at all study visits.

\section{Measures}

\section{Quantitative Assessment}

A brief baseline questionnaire assessed (1) sociodemographics, including age, race, ethnicity, sexual orientation, relationship status, education, and income; (2) health information, including current PrEP status, PrEP nonadherence over the previous two weeks, and STI history; (3) sexual history, including the number and types of sexual partners over the last three months, and condom use in the last 30 days; and (4) substance use history, including the types, frequency, and severity of substances used. The type of smartphone owned (i.e., Apple or Android) was also recorded for all participants. The assessment was comprised of all categorical variables. Responses were either write-in (e.g., age) or multiple choice; multiple choice questions directed participants to either select one response (e.g., sexual orientation) or to select all responses that apply (e.g., race). All quantitative data were collected and managed via the Research Electronic Data Capture (REDCap) system [16].

\section{Qualitative Interview}

Qualitative data were collected using a semi-structured interview guide, which included questions developed by two members of the study team (PC, RKR) in accordance with the Technology Acceptance Model (TAM) [17]. The TAM is a theoretical information technology framework that proposes that the acceptability and real-world adoption of a novel 
technology is primarily driven by its perceived usefulness and perceived ease of use. The interview guide was piloted among several study team members (PC, YM, GRG, MJB) prior to use with participants in order to confirm the clarity of all questions and probes. Interviews explored participants' experiences using the DPS during the study period, perceived barriers and facilitators to use of the technology, the ways in which participants integrated use of the DPS into their daily routines, and participants' willingness to use the technology long-term. Interviews were conducted by three members of the study team, two of whom are physicians with training in emergency medicine, general surgery, and substance use disorders (PRC, YM), and one of whom is a clinical psychologist (MJB).

\section{Analyses}

\section{Quantitative Data}

Descriptive statistics were calculated for selected variables from the quantitative assessment at baseline.

\section{Qualitative Data}

All interviews were digitally recorded and professionally transcribed. Transcripts were analyzed using applied thematic analysis $[18,19]$. Three members of the study team (GRG, YM, JN) independently reviewed all transcripts and iteratively generated an overarching coding framework for the identification of themes. Formal coding was completed by two study team members $(\mathrm{OB}, \mathrm{AB})$ who coded individually. The coders met after independently reviewing transcripts in increments of five, in order to compare coding and compare and correct data bracketing. Following each set of five transcripts, the coders paused and calculated a kappa score. If scores were less than 0.75, a third reviewer (PRC) met with the two coders to ensure that the coding schema was concordant prior to continuing the coding process. In aggregate coding was reviewed by several members of the study team (PRC, GRG, OB, AB, GG) and salient content areas were extracted and discussed. Qualitative analyses were facilitated by NVivo software [20]. The coding team consisted of the study principal investigator, who designed the study and qualitative interview guide, and six study team members, all of who were trained in qualitative interview and analysis techniques, and of whom only two had human subjects contact.

\section{Results}

Of 42 individuals pre-screened over the study period, 17 individuals attended a screening visit to undergo formal eligibility screening, of whom 16 were eligible and enrolled.
Two participants were lost to follow-up; however, one participant re-engaged in the study approximately five months later and completed a qualitative interview. Therefore, a total of 15 individuals completed the study. Sociodemographics for the study completers are reported in Table 1 . The median age was 32 years old (range 24-49). Participants primarily self-identified as White (67\%), not Hispanic or Latino (73\%), homosexual or gay (87\%). Most were taking PrEP at the time of enrollment (93\%) and had completed college (86.7\%). The most frequently self-reported substance used among participants was alcohol (93\%) followed by marijuana $(66.7 \%)$.

Four major content areas emerged from qualitative interviews surrounding the feasibility and acceptability of the DPS: (1) overall experience with DPS technology; (2) engagement with the DPS; (3) barriers to use and willingness to engage with the DPS long-term; and (4) suggestions for improvements and further development of the technology.

\section{Overall Experience with DPS Technology}

Following 90 days of DPS use, most participants reported that the technology was novel and valuable. Many noted that having access to their personal adherence data was a useful aspect of the system, as it increased their perceived accountability for adhering to PrEP and provided them with an ondemand record of their PrEP use. Participants did not experience any major side effects from taking PrEP and largely felt comfortable using the DPS from a safety perspective. Even when participants did not perceive personal lapses in their PrEP adherence, they still reported that using the technology was not a major inconvenience and could be useful for others. In instances where the DPS did not function as expected (e.g., technology-based issues), most participants were able to troubleshoot with relative ease; however, some participants experienced difficulty resolving such problems.

I think the digital pill is pretty awesome to be able to track when you're taking PrEP. You have a record of when you've taken it... When I take it without the machine, sometimes I wonder whether or not I've taken it, so it's good to kind of keep track for my records when I've taken it and [at] what time. (Age 28) My thoughts about it after taking it for three months, I guess you could say I feel like it's completely safe. I didn't have any adverse side effects. I felt very comfortable using the technology. In terms of the Reader, it was pretty easy to use. I wished it were a little bit smaller, but I know that people are working on that to make the device smaller, which is great. (Age 29) From my perspective, as long as everything was going fine, then it was great. A couple of areas where there 
Table 1 Sociodemographics and behavioral risks of study completers

\begin{tabular}{lll}
\hline Variable & $\begin{array}{l}\text { Sample } \\
(\mathrm{n}=15)\end{array}$ \\
\cline { 2 - 3 } & $\mathrm{n}$ & $\%$ \\
\hline
\end{tabular}

Age (in years)

Median (IQR)

Range

Race

White

Black

Asian

More than one race

Other

Ethnicity

Not Hispanic or Latino

Hispanic or Latino

Sexual orientation

Homosexual or gay

Bisexual

Relationship status

Single

Domestic partnership

Married

Separated

Education

Some college

College degree

Graduate degree/professional

Annual income

Less than $\$ 6,000$

$\$ 12,000$ to $\$ 17,999$

$\$ 30,000$ to $\$ 59,999$

$\$ 60,000$ or more

Prescribed PrEP

Yes

No

Nonadherent to PrEP in prior 2 weeks (self-report) ${ }^{\mathrm{a}}$

Yes

No

Ever had an STI

Yes

No

STIs in prior 12 months

Chlamydia

Gonorrhea

Syphilis

None

Number of sexual partners in past 3 months

Median (IQR)

Range

Condom use in past 30 days

Never

$10-67$
Table 1 (continued)

\begin{tabular}{lll}
\hline Variable & \multicolumn{2}{l}{$\begin{array}{l}\text { Sample } \\
(\mathrm{n}=15)\end{array}$} \\
\cline { 2 - 3 } & $\mathrm{n}$ & $\%$ \\
\hline Almost never & 2 & 13 \\
Sometimes & 1 & 7 \\
Almost every time & 4 & 27 \\
Every time & 3 & 20 \\
Not applicable & 1 & 7 \\
Non-medical use of stimulants & & \\
Yes & 7 & 47 \\
No & 8 & 53 \\
Frequency of stimulant use in past 30 days & & \\
Never & 6 & 40 \\
One or two times & 7 & 47 \\
About once a week & 1 & 7 \\
Several times a week & 1 & 7 \\
Other substances used & & \\
Alcohol & 14 & 93 \\
Marijuana & 10 & 67 \\
Hallucinogens & 4 & 27 \\
Other (poppers, amyl nitrate) & 7 & 47 \\
Type of smartphone owned & & \\
Apple & 9 & 60 \\
Android & 6 & 40 \\
\hline
\end{tabular}

${ }^{a}$ Defined as missing $>2$ doses of PrEP over the past two weeks. The denominator for this variable is 14 , as 14 participants had already been prescribed PrEP, and one had not

[was] real trouble... someone less technologically capable would not have been able to deal with it. On top of that, [the DPS] did not interact very well with some of the normal technology things that people already do in their day-to-day life, such as upgrade their phone [or] get a new device. That was not straightforward to be able to move it over, or even swapping the Reader itself was a large hassle. (Age 32)

\section{Engagement with the DPS}

Participants reported that, while they found the DPS useful overall, they did not significantly engage with the system outside of using it to record their PrEP adherence. Among those who did engage with the smartphone app, they generally reported that the app was straightforward and easy to use. Many participants liked the calendar view within the app in particular, as it provided them with access to their prior ingestion history. Participants also perceived the in-app notifications that indicated the successful detection of their ingestions to be helpful for confirming that they had correctly operated the DPS. Overall, however, engagement with the app was limited 
to the calendar view; if the technology was working properly, most participants devoted little time toward engaging with other app features.

I will say that was a useful part of the whole thing, being able to really track that I took [PrEP]. [Now] I'll have to go back to counting them or something like that, trying to find ways to figure out if I actually did it or not. (Age 32)

I opened the app when I took the pills. Basically, that's it. I didn't really use the app other than to check I took the pill. (Age 32)

Many participants were able to easily integrate the use of the DPS into their daily routines. They discussed a number of strategies for doing so, including taking the digital pill first thing in the morning, while brushing their teeth, simultaneously with other medications, or at mealtimes. One participant reported that the size of the Reader and the requirement to wear it while taking their digital pill was itself a daily visual reminder for maintaining their PrEP adherence. Most participants reported taking their PrEP at home and thus did not consider the use of the DPS to be a potential violation of their personal privacy. Most participants did not report major challenges associated with integrating the use of the new technology into their lives, and described the operation of the DPS as relatively equivalent to their existing medication-taking behaviors.

For the most part, it didn't add more than a minute to my morning routine. I'd get out of the shower, get dressed, throw it on, and then as I was making tea and food and stuff, I would take it and then be done with it. It didn't really add much time to my day. (Age 40)

However, some participants did report that the operation of the DPS, especially the Reader, represented a barrier to consistent use of the technology. This was described as particularly acute when participants' regular routines were already disrupted, such as while on vacation or when running late for work. One participant noted difficulty keeping track of all the DPS components (e.g., Reader and charger) due to changes in his personal and professional life that occurred during the study period.

Just the cumbersome nature of it. If you're goin' away for a weekend, it's one extra thing. Not only do you have to remember to pack the pills, but then you have to remember to pack [the Reader], too. It just kind of is one extra thing, I think is the hardest part of it, and it's a little bulky. (Age 40)

\section{Barriers to Use and Willingness to Engage with the DPS Long-Term}

Participants reported mixed feelings when asked about their willingness to use the DPS over a long-term period. Most noted that the system had clear value for improving PrEP adherence, but felt that one or more of the following barriers impacted their interest in long-term use: (1) technology-related issues with the app, Reader, and/or charger, (2) design-related issues with the Reader and/or charger, and (3) a perceived lack of personal PrEP adherence challenges.

Issues with the app, Reader, and charger largely centered around user-friendliness. While most participants noted that technology-related issues were addressed with relative ease, as discussed above, they impeded some participants' interest in long-term use of the DPS. Notably, however, participants generally trusted the DPS to safeguard their personal information and did not report data privacy as a major barrier to using the system.

If $I$ had to take a digital medication for the rest of my life, I would be okay with it. It's more just making sure that the technology fits with my life... the machine should be working for me. I shouldn't be working for the machine. (Age 32)

I don't think it was easy to operate and understand. The app was pretty straightforward. That was easy enough, but the Reader, the blinking lights didn't always make sense. It would blink red when it was 70 percent charged, and then the app sometimes would tell me it was low in charge, and then show me that it was 70 or 80 percent chargin'... The charger was poor quality, so I had to switch cables to try to charge it. Sometimes... I wouldn't know if it was charging or not, so it could definitely be easier. (Age 38)

As for design-related issues that impacted individuals' reported willingness to use the DPS long-term, the wearable Reader was the clear breakpoint in the technology. Participants overwhelmingly expressed a desire for a Reader designed to be more discreet and less bulky.

The hardest thing is the multi-part. If all I needed was my phone and the pill, I think that would be a no-brainer, "cause everyone has their phone on them every day now, no matter what, and the pill you have to take whether you're taking [digitized] PrEP or just the traditional little blue pill without the casing and the chip... When you add the Reader, that's a whole other set of moving parts, "cause then the Reader comes with the charger, and the charger comes with a cable. All of that is very annoying. I probably wouldn't wanna do it. (Age 38) 
Finally, a lack of personal perceived adherence issues limited some participants' interest in long-term use of the DPS, even if they were otherwise accepting of the technology. Many of these participants still noted that the system would be beneficial for individuals who were currently experiencing adherence-related challenges.

If people literally have a difficult time adhering to a daily regimen or time to take their pills, getting reminders and being held accountable via a digital recording system I think could be good... I think, overall, having your intake monitored would be beneficial for people [for whom] either it's extremely critical that they take the pill every day or just as a reminder to not forget. (Age 33)

\section{Suggestions for Improvements and Further Development of Technology}

Overall, participants found the DPS helpful for measuring their adherence behavior, but suggested several enhancements to the core technological components of the system, as well as to its messaging features, that would improve their user experience. Technological suggestions centered around design factors and DPS usability, including a desire for improving the portability of the Reader and charger by making it more compact and discreet. Many proposed a smaller device that could be attached to one's phone, in lieu of the current Reader that is worn on lanyard around the neck. A multi-purpose, integrated wristband or watch that could function as a Reader was another common suggestion for making the device itself less conspicuous.

If there's an advancement in the Reader technologyput a thin thing on your phone or something for the Reader instead of having to have a whole separate thing, then I think it would be even more seamless and quick... I just thought the strap could be a little bit more comfortable, but it wasn't bad. (Age 32)

If the signal strength was longer, I think a watch would be easier in the morning, or probably in the evening too. An armband kind of thing, so that you could still be doing stuff instead of standing motionless waiting for it to record. If it had to be right next to your skin... some joggers wear an elastic band around their chest to help monitor their heartbeats. Maybe that would have made it better for me...people could pick the format they thought might work for them. (Age 49)

Participants additionally made suggestions related to improving the programming of the smartphone app, in order to further streamline the user experience.

Keeping the app up to date and connected... would have to be fixed. Making that user experience better, keeping the data in such that I can see it regardless if I reset the device... I think there are still some technical bugs that need to be worked through. Those kinds of things would make a big difference. (Age 32)

As for the messaging components of the DPS, participants reported a desire for reminder messages and follow-up messages after missed doses. Participants also noted that having the option to customize standardized ingestion confirmation messages, reminder messages, and nonadherence follow-up messages - as well as the ability to opt in or out of certain messaging modules - would be helpful additions to the system, including for reasons related to personal privacy. Participants additionally reported that the lack of identification of PrEP as the medication of interest within the DPS was helpful for keeping their use of PrEP private. Key suggested customizations centered around the content, tone, and timing of DPS messages with an intent to provide contingent reinforcement around PrEP adherence, while also preventing the unwanted disclosure that the DPS was being utilized to measure PrEP ingestions. Additionally, participants suggested that DPS messages could include educational information related to PrEP, maintaining adherence, and behavior change more generally.

For instance, if I run for five days a week... my Fitbit will say, "Oh, good job. You did exercise for five days this week. That's great." You could add some more positive reinforcement, potentially... the longer the [adherence] streaks, the better. You might record what your longest streak is... to keep people motivated. (Age 32)

I think that "Ingestion detected" is vague enough so that way it makes sense to you, to the person that's taking it, but also someone else wouldn't know what it is... You could [also] choose what the notification would say. I think that would be really interesting... I could see for other people that wouldn't want it to be popping up on their phone in front of people, that they might prefer a thumbs up or a thumbs down. (Age 29)

\section{Discussion}

The effectiveness of once-daily oral PrEP for preventing HIV infection is contingent upon adherence [1,2]. Advances in measuring and evaluating personal adherence trends can provide patients with data on their adherence patterns and can equip healthcare providers with valuable information that may lead to interventions to support regular PrEP adherence. Digital pills are emerging as a potential electronic adherence tool that objectively verify PrEP ingestion events and can serve as a platform through which to deliver interventions based on adherence data. The present study demonstrates 
that MSM participating in real-world use of the DPS over 90 days are able to integrate the system into their personal routines, record PrEP adherence data, and are willing to engage and operate the technology on a daily basis. There were some concerns about the use of the wearable Reader, but participants were able to develop strategies to overcome these in the context of this study and consistently utilized the DPS. These findings are consistent with earlier qualitative work conducted by our research team around the perceived use and theoretical acceptability of the DPS among MSM with non-alcohol substance use [9].

We discovered several important facilitators associated with acceptable DPS operation, including the relative ease of integration into daily medication-taking behaviors, the ease of operating the technology, and the ability to access objective, personal adherence data on-demand. These features suggest that, even in its current iteration, the DPS can be utilized in a manner that is acceptable to MSM for PrEP adherence monitoring. The smartphone app was perceived to be the most important facilitator of the DPS components; participants viewed the design of the app as simple, straightforward, and easy to use, with the calendar view identified as its most engaging feature. Confirmation notifications within the app were perceived to be helpful for tracking ingestion events and as a tool for continued adherence motivation. Participants described referring to the app in order to check their past adherence, and reported anticipating upcoming ingestion events in the app while using the DPS. Finally, participants suggested that reminder messages be integrated into the app to further support PrEP adherence.

Participants clearly viewed the DPS as an integrated system - consisting of the digital pill, the Reader, and an app - that provides data visualization and feedback based on adherence, with the potential for enhancements via messaging modules. Future iterations of the DPS, and in particular the user-facing app interface, should be informed directly by such user experiences and preferences. Though future iterations of DPS may integrate cellular radios into the Reader to permit direct transmission of ingestion data without the use of a smartphone, this study suggests that data visualization is important to DPS users and should therefore remain a core component of the technology as it continues to develop.

Participants identified the use of an external wearable Reader as the most significant barrier to use of a DPS, and, while the overall feasibility of the DPS has been demonstrated across various disease states $[6,7,13,14]$, the use of a wearable device that is required to capture in-body radiofrequency signals from the DPS was challenging for some participants. The primary reported challenges related to the Reader included forgetting to charge it or not wearing it during digital pill ingestions. The Reader may therefore represent a barrier to adoption in some clinical settings, despite some qualitative feedback from the present study that some individuals may find the Reader to be a helpful component of the DPS. With the current inception of the DPS, deployments of the system outside the auspices of a clinical trial should be paired with training programs that teach use of the technology and address the Reader as a potential barrier to utilization [15]. Future technological advances may allow for more unintrusive wearable devices to replace the current lanyard-based Reader, or, alternatively, may integrate Reader technology into other electronic devices, like smartphones or wireless home routers increasing the usability of the system. Yet, the use of some form of a wearable Reader device will likely continue to be necessary into the near future. As such, techniques for circumventing this barrier, including those that help users integrate the use of the Reader into their daily routine, remain critically important; the practical strategies developed by participants in this study (e.g., storing the Reader in plain sight and using it as a visual reminder to take PrEP) represent useful suggestions. Future advances in signal boosting, as well as improved gastric energy harvesting [21] and miniaturized radio receivers, may contribute to the continued development of DPS technology and help to address some of the operational barriers that are associated with a larger wearable Reader.

Based on our qualitative findings, future iterations of the DPS should incorporate innovations in hardware as well as enhanced adherence supports. Hardware development updates should principally include miniaturizing the Reader and improving its battery life. Future investigations should also explore the integration of behavioral interventions in conjunction with the DPS in order to further optimize dayto-day use. In this investigation and in prior qualitative work among MSM [9], participants responded positively to the prospect of messaging modules linked to the DPS, and noted that integrated reminder messages would have the potential to help reinforce users' commitment to maintaining PrEP adherence. These modules could be simple text message systems that serve as reminders that can be triggered when nonadherence is detected by a DPS, or more in-depth bidirectional messages that center the conversation of PrEP adherence around objective measures of ingestion [22, 23]. Finally, text messaging that integrates DPS ingestion patterns with other contextual measures around adherence (e.g., smartphone usage or location information) may make adherence reminders more salient and personalized for individuals using such a system.

Future investigations should also explore the potential benefits of short-term use of the DPS. This investigation focused on experiences with the DPS and willingness to use it long-term, but measuring and supporting adherence in the short-term may additionally be a valuable application of the system. The DPS could also be utilized with patients beginning PrEP for the first time, with the technology packaged as part of a starter kit to help individuals learn about 
the basics of PrEP use and establish baseline adherence behaviors. Moreover, evidence-based behavioral interventions could be integrated into the DPS and implemented as a booster system for patients experiencing shorter-term periods of nonadherence. In light of the COVID-19 pandemic and its aftermath - particularly in the context of disruptions to mental health care access and treatment uptake, as well as substance use treatment [24] — strategies that support the successful HIV prevention in this vulnerable group will continue to be crucial.

This study has several limitations. First, the sample size was relatively small, and the majority of participants were White and well-educated. Experiences with the DPS may vary substantially across different racial groups and education levels; therefore, these findings may not be generalizable to all MSM with non-alcohol substance use. It is important that DPS technology be piloted among racial and ethnic groups who have traditionally experienced disparities related to PrEP access, uptake, and adherence. We have taken steps in our ongoing clinical trials to recruit and enroll a more diverse sample in order to capture a broader array of user experiences and to better understand the barriers that may exist in deploying the DPS among individuals who may be at the greatest risk for PrEP nonadherence. Second, individuals were excluded from participation if they did not own a smartphone; as such, disparities in access to technology is therefore highly relevant when considering the impact of a potential DPS-linked intervention on individuals with varying levels of technology access. Third, most participants in this formative investigation experienced mild to moderate substance use, and primarily used non-injection substances. Further research will need to explore the operational and individual-level barriers associated with utilizing DPS technology among individuals with significant substance use, including injection drugs, that may heighten HIV risk and impact PrEP adherence. Finally, the appeal of engaging with an innovative technology may have impacted participants' self-selection into the study, such that those with a greater interest in and comfort with technology may have been more likely to enroll and subsequently engage with the DPS throughout their participation in the study.

\section{Conclusion}

Overall, the DPS was feasible and acceptable for measuring real-time PrEP adherence in a sample of MSM with nonalcohol substance use. Participants were able to integrate the DPS into their daily lives and routines; however, certain aspects of the hardware need to be enhanced in order to optimize user experience of the technology. Future investigations should explore integrating behavioral components, such as adherence reinforcement messaging modules, into the DPS to augment adherence behavior.

Author Contributions PRC was the principal investigator, was responsible for the conceptualization and design of the study, and oversaw all methodological decisions and data collection. KHM, EWB, CO, and RKR contributed to the conceptualization and design of the study. GRG, MJB, and YM contributed to the study methodology, recruitment, and data collection. GRG, OB, GG, AB, JN, and YM conducted the qualitative analyses. MJB and GRG conducted data management and verification, with oversight from PRC. PRC, GRG, OB, GG, and $\mathrm{AB}$ drafted the manuscript, with contributions and editing from MCS, KHM, CO, and RKR. All authors reviewed and approved the final manuscript.

Funding This investigation was funded by an investigator-initiated research award from Gilead Sciences (ISR 17-1718).

Data Availability Not applicable.

Code Availability Not applicable.

\section{Declarations}

Conflict of interest PRC is funded by NIH K23DA044874, R44DA051106 and research funding from Gilead Sciences (ISR-171018), Hans and Mavis Lopater Psychosocial Foundation, Defense Advanced Research Programs Agency and The Bill and Melinda Gates Foundation. KHM and CO are funded by NIAID P30AI060354. EWB and RKR are funded by NIH R01DA047236.

Consent to Participate Informed consent was obtained from all individual participants included in the study.

Consent for Publication Not applicable.

Ethical Approval This study was performed in line with the principles of the Declaration of Helsinki. Approval was granted by the Fenway Community Health Institutional Review Board (protocol number 1087733)

\section{References}

1. Anderson PL, Glidden DV, Liu A, Buchbinder S, Lama JR, Guanira JV, et al. Emtricitabine-tenofovir exposure and preexposure prophylaxis efficacy in men who have sex with men. Sci Transl Med. 2012;4(151):151ra125.

2. Doblecki-Lewis S, Cohen S, Liu A. Clinical treatment options infectious diseases: update on PrEP implementation, adherence, and advances in delivery. Curr Treat Options Infect Dis. 2015;7(2):101-12.

3. Wood S, Gross R, Shea JA, Bauermeister JA, Franklin J, Petsis D, et al. Barriers and facilitators of PrEP adherence for young men and transgender women of color. AIDS Behav. 2019;23(10):2719-29.

4. Spinelli M, Haberer J, Chai P, Castillo-Mancilla J, Anderson P, Gandhi M. Approaches to objectively measure antiretroviral medication adherence and drive adherence interventions. Current HIV/ AIDS Rep. 2020 (in press). 
5. Bell KM, Haberer JE. Actionable adherence monitoring: technological methods to monitor and support adherence to antiretroviral therapy. Curr HIV/AIDS Rep. 2018;15(5):388-96.

6. Kamal S, Rosen MI, Lazar C, Siqueiros L, Wang Y, Daar ES, et al. Perceptions of people living with HIV and HIV healthcare providers on real-time measuring and monitoring of antiretroviral adherence using ingestible sensors: a qualitative study. AIDS Res Treat. 2020;1(2020):1-10.

7. Browne SH, Behzadi Y, Littlewort G. Let visuals tell the story: medication adherence in patients with type II diabetes captured by a novel ingestion sensor platform. JMIR mHealth uHealth. 2015;3(4):e108.

8. Grov C, Rendina HJ, John SA, Parsons JT. Determining the roles that club drugs, marijuana, and heavy drinking play in PrEP medication adherence among gay and bisexual men: implications for treatment and research. AIDS Behav. 2019;23(5):1277-86.

9. Chai PR, Goodman G, Bustamante M, Mendez L, Mohamed Y, Mayer KH, et al. Design and delivery of real-time adherence data to men who have sex with men using antiretroviral pre-exposure prophylaxis via an ingestible electronic sensor. AIDS Behav. 2020. https://doi.org/10.1007/s10461-020-03082-y.

10. Flores GP, Peace B, Carnes TC, Baumgartner SL, Buffkin DE Jr, Euliano NR, et al. Performance, reliability, usability, and safety of the ID-Cap system for ingestion event monitoring in healthy volunteers: a pilot study. Innov Clin Neurosci. 2016;13(9-10):12-9.

11. Camp C, Saberi P. Facilitators and barriers of 2-1-1 HIV preexposure prophylaxis. PLoS ONE. 2021;16(5):e0251917.

12. Hojilla JC, Marcus JL, Silverberg MJ, Hare CB, Herbers R, Hurley $\mathrm{L}$, et al. Early adopters of event-driven human immunodeficiency virus pre-exposure prophylaxis in a large healthcare system in San Francisco. Clin Infect Dis. 2020;71(10):2710-2.

13. Peters-Strickland T, Pestreich L, Hatch A, Rohatagi S, Baker RA, Docherty J, et al. Usability of a novel digital medicine system in adults with schizophrenia treated with sensor-embedded tablets of aripiprazole. NDT. 2016;12:2587-94.

14. Browne S, Peloquin C, Santillo F, Haubrich R, Muttera L, Moser $\mathrm{K}$, et al. Digitizing medicines for remote capture of oral medication adherence using co-encapsulation. Clin Pharmacol Ther. 2018;103(3):502-10.

15. Chai P, Bustamante M, Goodman G, Mohamed Y, Najarro J, Sullivan M, et al. A brief training program to support use of a digital pill system for medication adherence. JMIR Format Res. 2021;5(4):e26213.

16. Harris PA, Taylor R, Thielke R, Payne J, Gonzalez N, Conde JG. Research electronic data capture (REDCap) - a metadata-driven methodology and workflow process for providing translational research informatics support. J Biomed Inform. 2009;42(2):377-81.

17. Davis FD. Perceived usefulness, perceived ease of use, and user acceptance of information technology. MIS Q. 1989;13(3):319-40.

18. Braun V, Clarke V. Using thematic analysis in psychology. Qual Res Psychol. 2006;3(2):77-101.

19. Guest G, MacQueen K, Namey E. Applied thematic analysis. Inc.: SAGE Publications; 2011.

20. QSR International. NVivo software 2018

21. Nadeau P, El-Damak D, Glettig D, Kong YL, Mo S, Cleveland $\mathrm{C}$, et al. Prolonged energy harvesting for ingestible devices. Nat Biomed Eng. 2017;1(3):0022.

22. Chai PR, Mohamed Y, Goodman G, Bustamante MJ, Sullivan MC, Najarro J, et al. Development of a digital pill and respondent behavioral intervention (PrEPSteps) for HIV pre-exposure prophylaxis adherence among stimulant using men who have sex with men. Transl Behav Med 2021. https://doi.org/10.1093/tbm/ibab1 17.

23. Liu AY, Vittinghoff E, von Felten P, Rivet Amico K, Anderson PL, Lester R, et al. Randomized controlled trial of a mobile health intervention to promote retention and adherence to preexposure prophylaxis among young people at risk for human immunodeficiency virus: the EPIC study. Clin Infect Dis. 2019;68(12):2010-7.

24. Zvolensky MJ, Garey L, Rogers AH, Schmidt NB, Vujanovic AA, Storch EA, et al. Psychological, addictive, and health behavior implications of the COVID-19 pandemic. Behav Res Ther. 2020;134:103715.

Publisher's Note Springer Nature remains neutral with regard to jurisdictional claims in published maps and institutional affiliations. 